$10.2478 / \mathrm{gb}-2020-0005$

sciendo

\title{
Fern von Aleppo: Narrative Themenentfaltung im interkulturellen Kontext
}

\section{Eman Mohamed ABDELFATTAH SAID Dozentin Dr.; Universität Ain Shams in Kairo; E-Mail: eman.abdelfatah@alsun.asu.edu.eg}

\begin{abstract}
In "Fern von Aleppo", the Syrian author Faisal Hamdo, who left his home in 2014 and sought refuge in Germany, tells of his very personal integration experiences. The book represents a kind of intercultural communication. In his book, Faisal Hamdo, who sees himself as a "mediator between the worlds", tries to give the German reader answers to many questions regarding Syrian culture. From a text linguistic point of view, this book identifies the narrative development that seems to be tailored to the intercultural context. Accordingly, the present article raises the following questions: Does the structure of classic narration differ from the structure of narration in an intercultural context? Which intercultural information units are presented in the text? How are they embedded in the narrative text? Which constituents of the narrative structure are suitable for realizing intercultural communication? Which communicative functions do the constituents of the narrative structure fulfill in an intercultural context?
\end{abstract}

The contribution sets itself the goal of analyzing the narrative structure to investigate how intercultural communication comes about through narration, how the intercultural information units are integrated into the constituents of the classic narrative structure so that they fulfill their communicative function, and to developa suitable analysis model.

Keywords: Faisal Hamdo - narrative structure - narrative development of topics - intercultural communication - intercultural context- Fern von Aleppo - integration experiences 


\section{Einleitung}

In seinem Erstlingswerk Fern von Aleppo ${ }^{l}$ erzählt der syrische Autor Faisal Hamdo, der 2014 seine Heimat verlassen und in Deutschland Zuflucht gesucht hat, von seinen ganz persönlichen Integrationserlebnissen. Das Buch stellt eine Art interkulturelle Kommunikation dar. Der Autor (Ich-Erzähler), der der islamisch-arabisch-syrischen Kultur angehört, bringt dem deutschen Leser seine Erlebnisse in Deutschland und Erinnerungen aus seiner Heimat in deutscher Sprache näher und berichtet außerdem von mehreren Situationen, an denen Angehörige deutscher und arabischer Kultur beteiligt sind. Besonders auffällig ist dabei die interkulturelle Kompetenz des Autors, der durch seine Integrationsbereitschaft vermag, seine Kenntnis über die deutsche Kultur zu vertiefen und sie sich zu Eigen zu machen. In seinem Buch versucht Faisal Hamdo, der sich als „Mittler zwischen den Welten“ versteht, dem deutschen Leser Antwort auf viele offen stehende Fragen bezüglich der syrischen Kultur und zum Leben in Syrien bzw. im arabischen Raum zu geben.

Aus textlinguistischer Sicht lässt sich in diesem Buch die narrative Themenentfaltung identifizieren, die mehr oder weniger auf den interkulturellen Kontext zugeschnitten zu sein scheint. Dementsprechend stellt sich der vorliegende Beitrag folgende Fragen: Unterscheidet sich die Struktur der klassischen Narration von der Struktur der Narration im interkulturellen Kontext? Welche interkulturellen Informationseinheiten werden im Text präsentiert? Wie werden diese in den narrativen Text eingebettet? Welche Konstituenten der narrativen Struktur taugen zur Realisierung der interkulturellen Kommunikation? Welche kommunikativen Funktionen erfüllen die Konstituenten der narrativen Struktur im interkulturellen Kontext?

Der Beitrag geht von der linguistischen Forschungsrichtung der interkulturellen Kommunikation aus, die sich vor allem mit

${ }^{1}$ Hamdo, Faisal: Fern von Aleppo. Wie ich als Syrer in Deutschland lebe. Hamburg: Edition Körber, 2018. 
der Struktur und Funktion der als Kommunikationsmittel und Informationsvermittler dienenden sprachlichen Ausdrücke beschäftigt $^{2}$. Dabei setzt sich der Beitrag zum Ziel, durch die Analyse der narrativen Struktur zu erforschen, wie die interkulturelle Kommunikation durch Narration zustande kommt, wie die interkulturellen Informationseinheiten in die Konstituenten der klassischen narrativen Struktur integriert werden, damit sie ihre kommunikative Funktion erfüllen, und dazu ein Modell zu entwerfen.

\section{Theoretische Vorüberlegungen}

Da die Narration den Schwerpunkt des Beitrags bildet, muss dafür zunächst ein Begriff konstatiert werden. Narration bedeutet im weitesten Sinn Erzählen. Dabei ist unter Erzählen „die sprachliche Inszenierung" eines meist selbst erlebten Ereignisses zu verstehen. Charakteristisch dafür sind die Sequenzierung und die „Versetzung“, d. h. die Leser werden in die Zeit und an den Ort der Erzählung versetzt. ${ }^{3}$ Unter dem Begriff Erzählen bzw. Erzählung ist der Vorgang des Erzählens von dessen Produkt nicht zu trennen, während sich der Begriff Narration im engeren Sinn nur auf den Vorgang des Erzählens bezieht. ${ }^{4}$

Genauer ausgedrückt handelt es sich bei dem Buch Fern von Aleppo um die sogenannte Alltagserzählung (personal narrative), die als diese Art von Erzählung definiert werden kann,

${ }^{2}$ Vgl. Kiklewicz, Aleksander: Kategorien der interkulturellen Linguistik in systembezogener Auffassung. In: Földes, Csaba (Hrsg.): Interkulturelle Linguistik im Aufbruch. Das Verhältnis von Theorie, Empirie und Methode. Tübingen: Narr Francke Attempto (59-75), 2011, S. 64.

${ }^{3}$ Vgl. Streeck, Ulrich (Hrsg.): Erinnern, Agieren und Inszenieren. Enactments und szenische Darstellungen im therapeutischen Prozess. Göttingen: Vandenhoeck \& Ruprecht 2000, S. 182.

${ }^{4}$ Siehe. Schmidt-Haberkamp, Barbara: Narration. In: Ludger, Kühnhart u. Tilman, Mayer (Hrsg.): Bonner Enzyklopädie der Globalität. Wiesbaden: Springer (985-995), 2017, S. 985. 
die ,vergangene Erlebnisse“ zum Thema hat. ${ }^{5}$ Die Alltagserzählung zeichnet sich vor allem durch die narrative Themenentfaltung aus. ${ }^{6}$

Die Alltagserzählung in diesem Buch zeichnet sich überdies durch die retrospektive Darbietung eigener Erfahrungen aus. Die vom Autor selbst erlebten Handlungen haben den Vorteil, dass er die vollständige und detaillierte Ereignisstruktur von Anfang bis Ende vor Augen hat. Somit ist es ihm möglich, je nach Zielgruppe die erzählten Erlebnisse mit relevantem Hintergrundwissen zu versehen, damit die kommunikative Funktion der Erzählung erfüllt wird. Schütze (1976) schreibt der Erzählung die folgende kommunikative Funktion zu: „Die Grundfunktion von Erzählungen, Nichtbeteiligte an in sich relativ geschlossenen Ereigniskonstellationen erlebter Wirklichkeit, d. h. an Geschichten, vermittelt teilhaben zu lassen"7. Dabei spielt selbstverständlich der Autor (Ich-Erzähler) eine bedeutende Rolle. Er muss über narrative Kompetenz verfügen.

Nach Schütze (1976) besteht die sogenannte ,spezielle narrative Kompetenz" aus der Fähigkeit zur verständlichen Reihung der narrativen Sätze, zur kausalen Verknüpfung der Ereignisse und zur Bewertung und Andeutung der Ereignisse. ${ }^{8}$ Diese Fähigkeiten beziehen sich nicht zuletzt auf die Vertextung der Narration. Schütze (1976) setzt jedoch die primitive Fähigkeit zur retrospektiven Verarbeitung eigener sozialen Erfahrungen

5 Vgl. Streeck, Ulrich (Hrsg.): Erinnern, Agieren und Inszenieren. Enactments und szenische Darstellungen im therapeutischen Prozess. Göttingen: Vandenhoeck \& Ruprecht 2000, S. 182.

6 Vgl. Brinker, Klaus: Linguistische Textanalyse. Einführung in Grundbegriffe und Methoden. Berlin: Erich Schmidt Verlag 2018, S. 64.

7 Vgl. Schütze, Fritz: Zur soziologischen und linguistischen Analyse von Erzählungen. In G. Dux u. T. Luckmann (Hrsg.): Beiträge zur Wissenssoziologie - Beiträge zur Religionssoziologie (S. 7-41). Opladen: Westdt. Verl. 1976, S. 8.

${ }^{8}$ Ebenda, S. 19f. 
voraus, d. h. die Fähigkeit, die Erfahrungen zu kategorisieren, zu differenzieren, zu verallgemeinern, zu kombinieren, u.a. ${ }^{9}$

Zusammenfassend muss der Erzähler seine eigenen Erfahrungen in einen soziokulturellen Rahmen zusammensetzen, diesen in ein aktuelles soziokulturelles Problem einpassen sowie die Kompetenzen haben, die schon selbst erlebten Erfahrungen angemessen in Anspruch zu nehmen, indem er eine gute Erzählung strukturieren kann.

Beim interkulturellen Kontext, in dem Angehörige verschiedener Kulturen beteiligt sind, handelt es sich um die Interferenz verschiedener Kultursysteme. ${ }^{10}$ So wird der soziokulturelle Rahmen der Erzählung in ein aktuelles interkulturelles Problem oder einen interkulturellen Kommunikationsbereich eingebettet.

\section{Konstituenten der narrativen Struktur}

Zur Abgrenzung der narrativen Struktur des Textes bzw. der narrativen Themenentfaltung legt Labov großen Wert auf die chronologische Abfolge der Ereignisse. Er hat drei Voraussetzungen festgelegt, zu denen die chronologische Abfolge mindestens zweier Ereignisse sowie die Übereinstimmung der Satzfolge mit der chronologischen Reihenfolge gehören. ${ }^{11} \mathrm{Im}$ Gegensatz dazu lässt Brinker die chronologische Abfolge außer Acht und setzt stattdessen die Abgeschlossenheit und Ungewöhnlichkeit des erzählten Ereignisses voraus. ${ }^{12}$

9 Ebenda, S. 16.

${ }^{10}$ Vgl. Kiklewicz, Aleksander: Kategorien der interkulturellen Linguistik in systembezogener Auffassung. In: Földes, Csaba (Hrsg.): Interkulturelle Linguistik im Aufbruch. Das Verhältnis von Theorie, Empirie und Methode. Tübingen: Narr Francke Attempto (59-75), 2011, S. 61.

${ }^{11} \mathrm{Vgl}$. Martinez, Matias u. Scheffel, Michael: Einführung in die Erzähltheorie. München: C.H.Beck. 2012, S. 162.

${ }^{12}$ Vgl. Brinker, Klaus: Linguistische Textanalyse. Einführung in Grundbegriffe und Methoden. Berlin: Erich Schmidt Verlag 2018, S. 66. 
Nach Labov/Waletzky ${ }^{13}$ (1973) lässt sich der narrative Text in sechs Konstituenten bzw. Phasen gliedern, die in einer bestimmten Abfolge vorkommen müssen. Diese sind: 1) „Abstract“ oder Zusammenfassung derganzen Geschichte, 2) Orientierende Angaben zu Zeit und Ort der Handlung und den handelnden Personen, 3) Komplikation oder die Darstellung des Handlungsknotens, 4) die Auflösung der Komplikation, 5) „Coda“ oder das Ende der Erzählung, in dem der Leser auf die „Schlussfolgerung" aufmerksam gemacht und wieder aus der Erzählwelt zurückgeholt wird, und 6) Evaluation, d. h. die Mittel, die vom Erzähler zur Einschätzung des Erzählten in alle anderen Phasen eingebaut werden. ${ }^{14}$

Labov zählt Wiederholungen, Vergleiche mit weiteren eventuellen Handlungen, Verdichtung durch Einbettung paralleler Handlungen, und Erklärungen zu den wichtigsten Evaluationsmitteln in der narrativen Struktur. ${ }^{15}$

Das Modell von Labov/Waletzky (1973) legt großen Wert auf die evaluativen Mittel im Text, da sie die kommunikative Funktion einer beliebigen Erzählung erfüllen, auch wenn es sich um einen fiktionalen Text handelt. Durch die evaluativen Elemente stellt der Autor den erforderlichen Kontakt zu seinen Lesern her und vermittelt ihnen die jeweilige Botschaft. ${ }^{16}$ Die evaluativen Mittel, die die eigenen „Bewertungen“ und „Deutungen" des Erzählers zum Ausdruck bringen, dienen außerdem zur Einbettung der erzählten vergangenen Erlebnisse und

${ }^{13}$ Siehe. Labov, William u. Joshua Waletzky: Erzählanalyse. mündliche Versionen persönlicher Erfahrung. In: Ihwe, Jens (Hrsg.): Literaturwissenschaft und Linguistik, Bd. 2. Frankfurt: Athenäum (78-126), 1973.

${ }^{14}$ Vgl. Martinez, Matias u. Scheffel, Michael: Einführung in die Erzähltheorie. München: C.H.Beck. 2012, S. 163.

${ }^{15}$ Ebenda.

${ }^{16}$ Ebenda, S.165. 
eigenen Erfahrungen in das passende aktuelle soziokulturelle bzw. interkulturelle Problem. ${ }^{17}$

Trotz der Kritik findet das Modell von Labov/Waletzky (1973) für die textlinguistische, themaorientierte Analyse der narrativen Struktur bzw. der Alltagserzählung noch immer große Anerkennung. ${ }^{18}$

Brinker (2018) kritisiert jedoch das Modell von Labov/ Waletzky (1973). Er unterscheidet zwischen der thematischen Grundstruktur, die von Labov/Waletzky standardisiert wurde sowie der realisierten thematischen Struktur und meint, dass die Realisierung der narrativen Themenentfaltung die Abweichung von der von Labov festgelegten Abfolge der Konstituenten benötigen könnte, damit die intendierte kommunikative Funktion des narrativen Textes, der er eine besondere Bedeutung zukommen lässt, erfüllt werden kann. Das kommunikationsorientierte von Brinker modifizierte Modell bezeichnet drei Funktionen für die Elemente narrativer Themenentfaltung, nämlich Situierung (Ort und Zeit), Präsentation (Komplikation und Auflösung) und Resümee (Coda). Zu Evaluationen können auch nach Brinker bewertende Elemente in alle Konstituenten der narrativen Struktur eingefügt werden. ${ }^{19}$

In diesem Beitrag wird die Kritik von Brinker in Betracht gezogen und die Ansicht vertreten, dass in der realisierten thematischen Grundstruktur eines narrativen Textes die festgelegte Abfolge der Bestandteile nicht eingehalten werden muss.

${ }^{17}$ Vgl. Schütze, Fritz: Zur soziologischen und linguistischen Analyse von Erzählungen. In G. Dux u. T. Luckmann (Hrsg.): Beiträge zur Wissenssoziologie - Beiträge zur Religionssoziologie (S. 7-41). Opladen: Westdt. Verl. 1976, S. 13.

${ }^{18}$ Vgl. Brinker, Klaus: Linguistische Textanalyse. Einführung in Grundbegriffe und Methoden. Berlin: Erich Schmidt Verlag 2018, S. 65.

${ }^{19}$ Vgl. Brinker, Klaus: Linguistische Textanalyse. Einführung in Grundbegriffe und Methoden. Berlin: Erich Schmidt Verlag 2018, S. 64. 


\section{Das integrierte Analysemodell}

Das Modell, das zur Analyse der narrativen Struktur im interkulturellen Kontext zu verwenden ist, muss neben den linguistischen Aspekten auch die interkulturellen Aspekte in Betracht ziehen. Deswegen bietet der vorliegende Beitrag ein integriertes Modell an, das das modifizierte Modell von Brinker (2018), das zur Analyse narrativer Strukturen geeignet ist, mit dem Modell von Kiklewicz (2011), das die interkulturelle Kommunikation aus linguistischer Perspektive betrachtet, vereint.

Das Modell von Kiklewicz (2011) determiniert acht Aspekte zur linguistischen Betrachtung einer interkulturellen Kommunikation. Diese $\operatorname{sind}^{20}$ :

1) Kommunikationsbereich: Die allgemeine Sphäre, in der sich die Kommunikation abspielt und ihre kommunikativen Funktionen zu erfüllen hat, z. B. Medizin, Politik, Religion, Wirtschaft, Alltagsleben.

2) Kommunikationssituation: Damit werden Zeit, Ort und handelnde Personen gemeint. Sie ist im Brinkerschen Modell mit der Situierung der Sequenzen gleichzusetzen.

3) Kommunikationsträger: Der Sender und der Empfänger der Informationen innerhalb der Kommunikation.

4) Die Mitteilung: Die Situation bildet den Hauptteil der Kommunikation. Damit wird im Brinkerschen Modell die Präsentation gemeint.

5) Der Code: Das verbale oder nonverbale „Zeichensystem“, in dem die Mitteilungen erzeugt werden.

6) Interaktion: Kommunikative Handlungen in Form von verschiedenen Sprechakten, z. B. Ratschlag, Aufforderung, Verbot, usw.

${ }^{20}$ Vgl. Kiklewicz, Aleksander: Kategorien der interkulturellen Linguistik in systembezogener Auffassung. In: Földes, Csaba (Hrsg.): Interkulturelle Linguistik im Aufbruch. Das Verhältnis von Theorie, Empirie und Methode. Tübingen: Narr Francke Attempto (59-75), 2011, S. 62. 
7) Relation: Damit wird die Beziehung zwischen dem Sender und dem Rezipienten gemeint. Sie bezeichnet auch die Kommunikationsstimmung feindlich, fremd, usw.

8) Die Konvention: Darunter ist das Weltbild oder die kulturelle Kompetenz der Kommunikationspartner verschiedener Kulturen zu verstehen.

Von ihnen werden lediglich die Aspekte mitbetrachtet, die für die Analyse der narrativen Struktur relevant sind. Im narrativen Text, der einen interkulturellen Kontext zeigt, geht es eher um Mitteilungen, die aufeinander folgen, voneinander abhängen und gemeinsam die interkulturelle Kommunikation zustande bringen. Das integrierte Modell kombiniert die von Brinker festgelegten narrativen Funktionen der verschiedenen Konstituenten (Situierung, Präsentation und Evaluation) mit deren interkulturellen Funktionen, so spricht man von präsentierenden Mitteilungen und evaluierenden Mitteilungen. Dementsprechend präsentiert die Mitteilung in der erzählten interkulturellen Situation die Komplikation und deren Auflösung, oder evaluiert die interkulturelle Situation bzw. andere Mitteilungen. Die Situierung bildet einen Berührungspunkt beider Modelle, jedoch werden dazu die Kommunikationsträger und -stimmung (Relation) gezählt, da im narrativen Text, in dem die interkulturelle Kommunikation im Mittelpunkt steht, die Kommunikationsträger gelegentlich zur gleichen Zeit die handelnden Personen der Erzählung sind.

Auch der Kommunikationsbereich ist für das Verständnis verschiedener interkultureller Mitteilungen entscheidend. Der Kommunikationsbereich stellt die aktuelle interkulturelle Problemkonstellation dar, in der alle Erlebnisse und Ereignisse kontextualisiert werden.

Schließlich wird auch das Brinkersche Resümee betrachtet, dem neben seiner klassischen Funktion in der narrativen Struktur auch eine andere interkulturelle Funktion zugeschrieben wird, nämlich die Zusammenfassung und Kommentierung 
der erzählten interkulturellen Mitteilungen sowie die Aufklärung der Leser über die kulturellen Unterschiede im behandelten Kommunikationsbereich.

Davon ausgehend basiert das integrierte Modell, das zur Analyse der narrativen Struktur im interkulturellen Kontext aus kommunikativ-funktionaler Sicht zu verwenden ist, auf folgenden Konstituenten: Kommunikationsbereich, Situierung (Ort, Zeit, Kommunikationsträger und-stimmung), präsentierende Mitteilung (Präsentation bzw. Komplikation und Auflösung), evaluierende Mitteilung (Evaluationen und Bewertungen), Resümee.

\section{Narrative Struktur in Fern von Aleppo}

Das Buch Fern von Aleppo besteht aus mehreren Erzählungen. Jede Geschichte thematisiert ein eigenes interkulturelles Problem und bildet einen eigenständigen narrativen Text. Im Folgenden werden mittels des neuen integrierten Modells die narrativen Strukturen einiger Erzählungen analysiert.

\section{Als mein Opa zum König wurde}

Kommunikationsbereich: Seniorenleben

Situierung: In einem Seniorenheim in Hamburg führen der Erzähler, der dort aushilft, und eine alte Bewohnerin des Seniorenheims an einem kalten aber sonnigen Tag im Februar 2015 ein Gespräch. Die Kommunikation spielt sich zwar auf neutraldienstlicher Basis, aber in freundlicher Stimmung ab.

Präsentierende Mitteilung: Das Gespräch stellt die Komplikation dar. Die alte Frau ist deprimiert, weil ihre Tochter sie nicht besuchen kommt. Der Erzähler versucht sie zu trösten, indem er ihr vorschlägt, mit ihr an der frischen Luft spazieren zu gehen. Ihre Laune hebt sich umgehend. 
Evaluierende Mitteilung 1: Diese Mitteilung wurde von der präsentierenden Mitteilung assoziiert. Sie zeigt einen Vergleich zwischen dem Leben der deutschen alten Frau, die sich in ihrem Alter einsam fühlt, in einem Seniorenheim lebt, ihre Tochter vermisst und sich nach einem Spaziergang und Gesellschaft sehnt, und dem Leben seines syrischen Großvaters, der bis zu seinem Tod in einem eigenen Raum bei seinem Sohn wohnte und die Pflege seiner Kinder und Enkelkinder genoss.

Evaluierende Mitteilung 2: Sie wurde von der präsentierenden Mitteilung assoziiert. In dieser Mitteilung beschreibt der Autor sein eigenes Familienleben mit dem Großvater. Es ist eine uralte und selbstverständliche Konvention in der Familie des Autors bzw. in jeder anderen syrischen Familie, das Großelternteil bei sich einziehen zu lassen, um ihm bzw. ihr die benötigte Pflege und Fürsorge zukommen lassen zu können. Der Großvater wurde als König behandelt und von seiner Familie gepflegt, ohne dass er etwas dagegen hätte einwenden können. Zur großen Freude seiner Enkelkinder verlor er, trotz seiner Demenz nie die Lust, über seine Vergangenheit zu erzählen.

Resümee: Der syrische Erzähler findet am Ende Antwort auf seine Frage, warum das Alter in Deutschland gefürchtet wird. Er fasst in der Form eines kurzen Vergleichs zusammen, wie das Altsein aus Sicht der alten Menschen in Syrien und in Deutschland empfunden wird. Er bringt auch seine Verlegenheit zum Ausdruck wenn er darüber nachdenkt, wie für ihn als Syrer, der sein weiteres Leben in Deutschland verbringen will, sein Leben im Alter wohl aussehen mag.

Die Interkulturalität durchdringt alle Phasen der narrativen Struktur. Der narrative Text hat das Seniorenleben in beiden Kulturen zum Thema, indem jede Mitteilung eine vollständige interkulturelle Informationseinheit bildet. Die interkulturellen Eigenschaften lassen sich in der Situierung des narrativen Textes dadurch erkennen, dass die handelnden Personen verschiedenen 
Kulturen angehören. Die erzählte Sequenz handelt von einem Gespräch zwischen zwei Kommunikationspartnern verschiedener Kulturen und zeigt ein interkulturelles Missverständnis seitens des Erzählers, der sich aufgrund seiner arabischen Kultur nicht vorstellen kann, dass die Tochter ihre Mutter nicht besuchen kommen kann. In den Evaluationen des Autors versucht er dem Leser das erzählte Missverständnis zu erklären, indem er an das Leben seines erschöpften und verwirrten Großvaters erinnert und dieses als Prototyp des Lebens der betagten Menschen in Syrien mit dem der deutschen betagten Menschen vergleicht. Die kommunikative Funktion der Erzählung ist dem deutschen Leser zu vermitteln, wie er als Syrer das Leben der Deutschen im Alter ansieht. Die evaluierenden Mitteilungen stehen in einer assoziierenden Beziehung zur präsentierenden Mitteilung.

\section{Die Ziege meiner Mutter}

Kommunikationsbereich: Das Verhältnis zu Zahlen, Daten und Terminen

Situierung: Ein Telefongespräch mit seiner Mutter, die mit seinem Vater im Exil lebt. Die handelnden Personen sind der Erzähler und seine Mutter. Ort und Zeit wurden nicht angegeben.

Präsentierende Mitteilung: Die Sequenz stellt ein Telefongespräch dar. Die Komplikation ist, dass die Mutter sich nicht wohlfühlt und weint, weil sie ihre Kinder vermisst. Als Auflösung der Situation gibt er ihr zum Spaß ein Rätsel über sein Alter auf, um ihr Trost zu spenden. Die Mutter gibt jedoch die falsche Antwort.

Evaluierende Mitteilung 1: Diese Mitteilung kommentiert die präsentierende Mitteilung, indem der Autor eine humorvolle Anmerkung macht, dass sich seine Mutter nicht für Daten interessiert, sondern sich eher an sie in Bezug auf die mit ihnen in 
Verbindung stehenden Ereignisse erinnert. Der Erzähler berichtet vergleichend, dass die Häuser in Syrien keine Hausnummern besitzen und deshalb keine Post zugestellt bekommen, während es in Deutschland gang und gäbe ist, dass man Briefe oder Päckchen nach Haus geliefert bekommt, wie es der Fall bei seinen Gasteltern ist. Seine Gastmutter kann - im Gegensatz zu seiner Mutter - mit Daten und Zahlen gut umgehen. Sie kann auch „What's-App“- Nachrichten schreiben und verschicken.

Evaluierende Mitteilung 2: Sie kommentiert die vorherige evaluierende Mitteilung 1, indem der Autor berichtet, dass er mehrere Terminkalender hat und sich freut, Post bekommen zu können.

Evaluierende Mitteilung 3: Sie wurde von der präsentierenden Mitteilung assoziiert. Der Erzähler zeigt sich darüber erstaunt, dass Kinder in Deutschland bei Eintritt ins Studium das Elternhaus lieber verlassen, obwohl sie in derselben Stadt studieren. Sie wohnen dann vorzugsweise in einer Wohngemeinschaft, während ihre eigenen Zimmer im Elternhaus leer stehen. Das findet er komisch und nicht nachvollziehbar. Ein Gespräch mit einem deutschen Arbeitskollegen ließ ihn zudem erfahren, dass Deutsche mittleren Alters, anstatt eigene Kinder zu haben, gern Hunde halten.

Evaluierende Mitteilung 4: Sie zeigt ein Gespräch zwischen dem Erzähler und der deutschen Frau über das Verhältnis der Deutschen zu ihren Hunden, die von ihnen als Familienmitglieder bzw. ihre Kinder betrachtet werden. Diese Mitteilung hängt von der vorherigen evaluierenden Mitteilung ab. Sie ergänzt trotz der neuen Situierung den Hauptgedanken der vorherigen Mitteilung über das Verhältnis der Deutschen zu den Tieren.

Evaluierende Mitteilung 5: Als dem Autor die besondere Beziehung der Deutschen zu ihren Haustieren, und dabei besonders Hunden, auffällt, denkt er spontan an die ähnliche Liebe seiner Mutter zu ihren Ziegen. 
Evaluierende Mitteilung 6: Das Einkaufen in klimatisierten Geschäften in Deutschland erinnert ihn an seine Mutter, die trotz der hohen Temperatur zu Fuß einkaufen gehen musste, um Geld zu sparen, um ihrem Sohn damit das Lernen auf seine Prüfungen zu ermöglichen.

Der Autor hat in diesem Fall auf die Angabe von Ort und Zeit verzichtet. Die Präsentation der Konversation des Autors mit seiner Mutter zeigt zwar keine interkulturellen Eigenschaften, jedoch assoziieren bei ihm drei Gedanken, die er in den folgenden evaluierenden Mitteilungen interkulturell zur Sprache bringt, nämlich das Verhältnis der Syrer und der Deutschen zu Zahlen, Daten und Terminen, Kommunikation per Post und das Verhältnis der Kinder zu ihrem Elternhaus. Die Präsentationsphase bildet hier fast nur einen Erzählanlass seiner nachstehenden interkulturellen Überlegungen und Vergleiche. Die evaluierende Mitteilung 1 kommentiert die präsentierende Mitteilung und bildet zusammen mit der evaluierenden Mitteilung 2 eine in sich abgeschlossene interkulturelle Informationseinheit. Die evaluierende Mitteilung 3 wurde von der präsentierenden Mitteilung assoziiert und führt zur evaluierenden Mitteilung 4, d. h. die evaluierenden Mitteilungen müssen nicht unbedingt von der präsentierenden Mitteilung abhängen. Sie können eine vorherige Mitteilung kommentieren oder ergänzen. Evaluierende Mitteilungen können auch situiert werden, wie im Fall der evaluierenden Mitteilung 4.

Ein weiterer Schwerpunkt (Beziehung zu Tieren) lässt sich in der evaluierenden Mitteilung (4) erkennen, die in der Form einer Konversation zwischen dem Autor und einer deutschen Frau über die Liebe der Deutschen zu Hunden formuliert wird. Die daran anschließende evaluierende Mitteilung des Autors (5) wird von der vorherigen evaluierenden Mitteilung (4) assoziiert, indem er sich an die Liebe seiner Mutter zu ihren Ziegen erinnert. Beide Mitteilungen ergänzen einander und bilden 
zusammen eine abgeschlossene interkulturelle Informationseinheit in Form eines Vergleichs. Im Gegensatz dazu steht die sechste evaluierende Mitteilung isoliert vom Kontext, d. h. sie wurde weder von der präsentierenden Mitteilung noch von einer vorherigen evaluierenden Mitteilung herbeigeführt. Die Erzählung verfügt über kein Resümee.

\section{Mein Männerleben als Jungfrau}

Kommunikationsbereich: Liebe, Geschlechtsverkehr und Freundschaft

Situierung: An einem warmen Morgen im März 2010 erhält der Autor einen Anruf von Evin, seiner kurdischen Kommilitonin, während er auf der Dachterrasse des Hauses die Sonne genießt.

Präsentierende Mitteilung: Der Autor erzählt von einem Telefongespräch mit Evin, seiner kurdischen Kommilitonin, die ihn zum Feiern des Newroz mit ihr zusammen einlädt. Es kommt zu Komplikationen als ihn seine Mutter mit einem Mädchen sprechen hört und wissen will, ob er sich in sie verliebt hat. Sie ist der Meinung, dass er sie unverzüglich zu heiraten hat, was der Autor nicht nachvollziehen kann. Als Auflösung der Situation leugnet der Autor, überhaupt in seine Kommilitonin verliebt zu sein.

Evaluierende Mitteilung 1: Sie kommentiert die präsentierende Mitteilung. Der Autor weist darauf hin, dass die syrischen Mütter ihre Kinder gern so schnell wie möglich verheiratet sehen wollen, bevor sie eine Sünde mit den geliebten Frauen begehen, und vergleicht diesen Umstand mit der Einstellung seiner deutschen Gastmutter, die es erlaubt, dass er ein Mädchen nach Hause einlädt und mit ihr schläft. Die Mitteilung zeigt ein Gespräch mit seiner Gastmutter über das muslimische HaramKonzept den vorehelichen sexuellen Beziehungen gegenüber. 
Zwischenresümee: Ein Kommentar vom Autor, der zusammenfasst, wie unterschiedlich man Liebe in Deutschland und Syrien erlebt und begreift.

Evaluierende Mitteilung 2: Zum Erklären und Beweisen des im Resümee zusammengefassten Fazits berichtet der Autor von einem weiteren Gespräch mit seinem Arbeitskollegen, Anton, der nicht glauben kann, dass er in seinem Alter noch keine einzige sexuelle Beziehung angefangen hat. Der Autor wird von seinen Kollegen „Jungfrau“ genannt.

Evaluierende Mitteilung 3: Diese Mitteilung kommentiert auch das Resümee. In dieser Mitteilung ist der Autor darauf angewiesen, sein Verständnis der Liebe durch seinen islamischen Glauben zu begründen bzw. zu verteidigen und seinen Stolz darauf zu zeigen, sich trotz der Integrationsversuche noch an seinem Glauben festhalten zu können.

Evaluierende Mitteilung 4: Hier zieht der Autor einen Vergleich zwischen der Sexualaufklärung im frühen Alter in Deutschland und der dagegen gesetzten Sexualunwissenheit unter den Pubertierenden in Syrien. Diese Mitteilung steht auch in einer kommentierenden Beziehung zum Resümee.

Das letzte Resümee: Eine erklärende Aussage vom Autor, die zusammenfassend bestätigt, dass er ohne Sex leben kann und stattdessen seiner Arbeit und Familie Priorität einräumt.

Die präsentierende Mitteilung bildet mit der evaluierenden Mitteilung 1 und dem Zwischenresümee eine in sich abgeschlossene Erzähleinheit. Die interkulturelle Behandlung erscheint zum ersten Mal im Vergleich in der evaluierenden Mitteilung, die neue Situierung benötigt. Die evaluierende Mitteilung steht in einer gegensätzlichen Beziehung zur präsentierenden Mitteilung. Das Resümee steht nicht am Ende der Erzählung, sondern in der Mitte und wurde durch weitere Handlungen und evaluierende Sequenzen bewiesen. Die 
evaluierenden Mitteilungen 2, 3 und 4 hängen vom Resümee $\mathrm{ab}$, wurden von ihm jedoch nicht assoziiert, sondern erfüllen eine bestimmte kommunikative Funktion, nämlich die Erklärung und Bestätigung der vom Autor dargebotenen interkulturellen Differenzen. Das Resümee sowie die damit verbundenen Mitteilungen stellen interkulturelle Differenzierungen dar, die inzwischen erklärt und kommentiert werden.

\section{Die Uhr meines Vaters}

Kommunikationsbereich: Atmosphäre innerhalb der Familie.

Situierung: Bei seinem Freund Jan. Die handelnden Personen sind Jan, Jans Eltern und der Autor.

Evaluierende Mitteilung 1: Die Anrede in der deutschen und arabischen Kultur fällt ihm ein, während er den Eltern seines Freundes vorgestellt wird, was er in der evaluierenden Mitteilung in der Form eines Vergleichs darbietet, der die kulturellen Unterschiede in diesem Zusammenhang erklärt.

Präsentierende Mitteilung: Mitten im Vorstellungsgespräch mit den Eltern seines Freundes erhält der Autor einen Anruf von seinem Vater aus dem Exil. Er fühlt sich peinlich berührt, kann aus moralischen Gründen das Gespräch nicht beenden und begnügt sich damit, seinem Vater mitzuteilen, dass er bei seinem Freund ist. Als Auflösung der Situation versteht der Vater die versteckte Botschaft und beendet von sich aus das Gespräch, indem er seine Grüße an den Freund seines Sohnes und seine Eltern ausrichtet.

Evaluierende Mitteilung 2: Die Mitteilung wurde von der präsentierenden Mitteilung assoziiert. Der Autor vergleicht zwischen seinem Vater, der als Bauarbeiter in Syrien gearbeitet hat und dem Vater seines Freundes, der in einem Bauamt in Deutschland tätig war. Wenn sein Vater gelegentlich am Monatsende 
nicht bezahlt wurde, schlug er aus Wut mit der Faust kräftig gegen die Wand, sodass seine Armbanduhr schließlich kaputt ging. Im Gegensatz dazu brauchte sich der deutsche Bauarbeiter in solchen Fällen nicht aufzuregen: Denn er war durch einen offiziellen Vertrag rechtlich geschützt.

Evaluierende Mitteilung 3: Sie wurde von der präsentierenden Mitteilung assoziiert. Es geht in dieser Mitteilung um den Respekt dem Vater gegenüber. Der Autor hat sich aus Respekt nicht getraut, das Gespräch mit seinem Vater zu beenden, wie er in der präsentierenden Mitteilung erklärt hat, während sein Freund seinem Vater so mutig widersprach.

Evaluierende Mitteilung 4: Sie ergänzt die evaluierende Mitteilung 3. Es geht um die Macht des syrischen Vaters innerhalb der Familie. Der Autor erzählt, wie sein Vater die Gesetze zu Hause bestimmte, die von allen Familienmitgliedern beachtet werden mussten und wie er die Rolle des Staatsanwaltes spielte.

Resümee: Am Ende stellt sich der Autor die Frage über das erstaunliche Lebenswissen und die Klugheit seines Vaters, der seine Kinder groß gezogen hatte und ihnen vieles beibringen konnte, obwohl er selbst nicht zur Schule gegangen war und kaum lesen konnte.

In der Situierung fehlen die Angaben zu Ort und Zeit. Die erste evaluierende Mitteilung durchdringt die Phase der Situierung. Die evaluierenden Mitteilungen werden in Form von interkulturellen Vergleichen gestaltet. Sie stehen in einer assoziierenden Beziehung zur präsentierenden Mitteilung. Die evaluierenden Mitteilung 3 und 4 bilden wiederum zusammen eine abgeschlossene interkulturelle Informationseinheit, indem sie inhaltlich miteinander verbunden sind. 


\section{Loriot für Araber}

\section{Kommunikationsbereich: Humor}

Situierung: An einem kalten Herbsttag tritt der Autor in Berlin einen neuen Job im großen Hausgarten einer typisch deutschen Familie an. Die handelnden Personen sind „Herr von Sowieso“, „Frau von Sowieso“ und der Autor.

Evaluierende Mitteilung 1: Die Mitteilung zeigt ein interkulturelles Problem. Der Autor ist erstaunt, als er die Präposition „von“ vor dem Familiennamen auf dem Schild liest. Er fragt sich, ob es sich hier um einen Titel wie „Beg“ handelt, der in der syrischen Gesellschaft Verwendung findet.

Präsentierende Mitteilung: Beim Kaffeetrinken im Wohnzimmer bemerkt der Autor viele gemalte Porträts und Gemälde an den Wänden. Dabei fällt ihm auf, dass ein Bild schief hängt und will es gerade rücken. „Herr von Sowieso“ gibt ihm daraufhin scherzhaft zu verstehen, dass er es nicht wie Loriot tun dürfe. Die Komplikation ist, dass der Autor den Begriff „Loriot“ nicht versteht. Noch peinlicher berührt ist er, als „Frau von Sowieso“ sich einschaltet und mit ihrem Mann ein langes Gespräch darüber führt, wie und warum er Loriot kennen solle. Es kommt zur Auflösung der Situation, als Herr und Frau „Sowieso“ den Autor über Loriot aufklären und ihm einige seiner Werke zeigen.

Evaluierende Mitteilung 2: Diese kommentiert die präsentierende Mitteilung. Sie erklärt, inwieweit sich die Deutschen für Humor interessieren, sodass mehrere vor allem politische satirische Sendungen -im Gegensatz zum syrischen Fernsehen- im Fernsehen und auf Youtube frei verfügbar sind. Die Mitteilung steht in einer kommentierenden Beziehung zur präsentierenden Mitteilung.

Evaluierende Mitteilung 3: Sie ergänzt die evaluierende Mitteilung 2, um eine vollständige interkulturelle Informationsein- 
heit zu bilden. Es geht hier um den Humor im offiziellen syrischen Fernsehen, in dem über Politik und Religion keine Witze gemacht werden dürfen. Diese Mitteilung steht in einer ergänzenden Beziehung zur evaluierenden Mitteilung 2.

Evaluierende Mitteilung 4: Sie wurde von der evaluierenden Mitteilung 3 assoziiert, indem der Autor vom Lachen über Religionen und Gläubige in der syrischen Gesellschaft weitererzählt. Sie steht in einer assoziierenden Beziehung zur evaluierenden Mitteilung 3.

Evaluierende Mitteilung 5: Sie wurde von der evaluierenden Mitteilung 4 assoziiert. Der Autor erzählt vom absurden syrischen Alltag, der schwarzen Humor auslöst. Als Beispiel dient ihm dazu eine Situation in einer öffentlichen Staatsbehörde.

Resümee: Der Autor fasst vergleichend zusammen, was den deutschen Humor vom syrischen Humor unterscheidet.

Die evaluierende Mitteilung 1 kommt in der Situierung vor. Sie zeigt, dass sich der Autor Gedanken über die deutsche Namenskultur macht. Die Komplikation in der präsentierenden Mitteilung kommt erst wegen eines kulturellen Missverständnisses vor und wird durch eine Aufklärung des Autors über die ungewusste kulturelle Angelegenheit aufgelöst. Die Mitteilungen 2 und 3 bilden zusammen eine abgeschlossene interkulturelle Informationseinheit in der Form eines Vergleichs. Die Mitteilung 4 ist von der vorherigen Mitteilung 3 assoziiert. Dergleichen wurde die Mitteilung 5 assoziiert.

Bemerkenswert in dieser Erzählung ist, dass sich die evaluierenden Mitteilungen verketten, indem jede von der vorherigen abhängig ist. Das Resümee behandelt die kulturellen Unterschiede in Bezug auf Humor in der deutschen und syrischen Gesellschaft. 


\section{Fazit}

Der narrative Text, der einen interkulturellen Kontext aufweist, besteht aus einer Zahl von Mitteilungen, denen zur Realisierung der interkulturellen Kommunikation, bestimmte Funktionen zugewiesen werden, nämlich Situierung, Präsentation, Evaluierung oder Resümieren. Der Kommunikationsbereich spielt dabei eine bedeutende Rolle, da die kommunikative Funktion verschiedener Mitteilungen nur in Bezug auf den jeweiligen Kommunikationsbereich zu determinieren ist.

Die narrative Struktur im interkulturellen Kontext weicht nicht von der klassischen narrativen Struktur ab, zeigt jedoch keine feste Struktur. In manchen Fällen fehlen einige Konstituenten wie z. B. das Resümee, oder es treten umgekehrt weitere Konstituenten wie z. B. ein Zwischenresümee auf.

Im narrativen Text, der einen interkulturellen Kontext aufweist, handelt es sich nicht um Ereignisse bestimmter chronologischer Abfolge, wie Labov meint, sondern um vereinzelte abgeschlossene Ereignisse bzw. interkulturelle Situationen, die durch den gemeinsamen Kommunikationsbereich miteinander verbunden sind.

Die präsentierenden Mitteilungen erweisen sich als Sequenzen mit einfachen abgeschlossenen Ereignissen, in denen die Komplikation und Auflösung auseinanderzuhalten sind. Diese präsentierenden Mitteilungen stehen - im Gegensatz zur klassischen narrativen Struktur - nicht im Mittelpunkt der Erzählung. Im interkulturellen Kontext fungieren die in den präsentierenden Mitteilungen erzählten Ereignisse nur als Einführung oder Sprechanlass zu den mit interkulturellen Informationen aufgeladenen evaluierenden Mitteilungen des Autors, die sich wiederum als der Kern des interkulturellen Kontextes verstehen.

Die evaluierenden Mitteilungen werden nicht nur in die präsentierende Mitteilungen eingebettet, sondern können auch ganz am Anfang in die Situierung oder ganz am Ende in das 
Resümee hinzugefügt werden, indem sie es bestätigen, erklären, beweisen oder kommentieren.

Evaluierende Mitteilungen können situiert werden und können auch Sequenzen zeigen, die - im Gegensatz zu den präsentierenden Mitteilungen - keine Komplikationen und Auflösungen benötigen. AufGrund der evaluierenden kommunikativen Funktion dienen diese Sequenzen verschiedenen Zwecken wie Erklären, Beweisen und Vergleichen.

Das Ziel der narrativen Struktur im interkulturellen Kontext ist, dass dem Leser interkulturelle Informationen vermittelt werden. Dementsprechend bildet jede evaluierende Mitteilung eine in sich abgeschlossene interkulturelle Informationseinheit bzw. vereinigt sich ansonsten mit einer anderen evaluierenden Mitteilung, umeineabgeschlosseneinterkulturelleInformationseinheit zu bilden. Es kann auch so weit gehen, dass die einander ergänzenden evaluierenden Mitteilungen isoliert von der präsentierenden Mitteilung einen neu bestehenden selbstständigen Aufbau mit einem neuen interkulturellen Schwerpunkt zusammenbilden.

Im narrativen Text müssen sich die Mitteilungen eines ein und demselben Kommunikationsbereichs aufeinander beziehen. Zwischen ihnen herrschen folgende Arten von Beziehungen: 1) eine assoziierende Beziehung, in der die eine Mitteilung die andere hervorruft, wobei es zu einem neuen Thema kommen kann, 2) eine kommentierende Beziehung, in der die eine Mitteilung die andere kommentiert, 3) eine ergänzende Beziehung, in der die eine Mitteilung die andere ergänzt, um eine in sich abgeschlossene interkulturelle Informationseinheit zu bilden, 4) eine gegensätzliche Beziehung, in der die zwei Mitteilungen gegensätzliche Inhalte zeigen. 